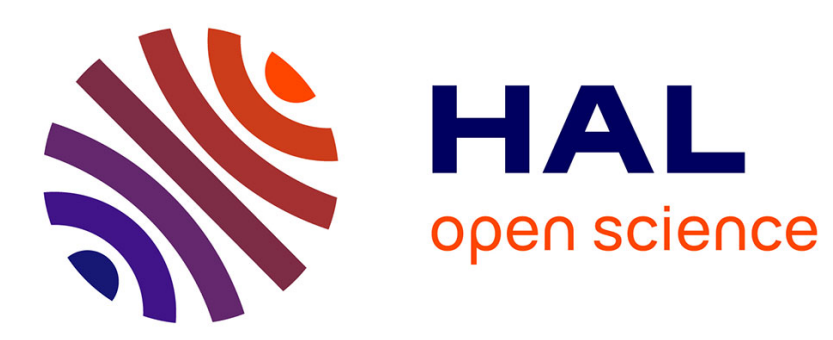

\title{
Dispositif automatique de commande de balayage et de mesure de la longueur d'onde pour spectromètre
}

\author{
J. Bonnet, L. Soonckindt, L. Lassabatère
}

\section{To cite this version:}

J. Bonnet, L. Soonckindt, L. Lassabatère. Dispositif automatique de commande de balayage et de mesure de la longueur d'onde pour spectromètre. Revue de Physique Appliquée, 1976, 11 (1), pp.175179. 10.1051/rphysap:01976001101017500 . jpa-00243962

\section{HAL Id: jpa-00243962 https://hal.science/jpa-00243962}

Submitted on 1 Jan 1976

HAL is a multi-disciplinary open access archive for the deposit and dissemination of scientific research documents, whether they are published or not. The documents may come from teaching and research institutions in France or abroad, or from public or private research centers.
L'archive ouverte pluridisciplinaire HAL, est destinée au dépôt et à la diffusion de documents scientifiques de niveau recherche, publiés ou non, émanant des établissements d'enseignement et de recherche français ou étrangers, des laboratoires publics ou privés. 


\title{
DISPOSITIF AUTOMATIQUE DE COMMANDE DE BALAYAGE ET DE MESURE DE LA LONGUEUR D'ONDE POUR SPECTROMÈTRE
}

\author{
J. BONNET, L. SOONCKINDT et L. LASSABATÈRE \\ Université des Sciences et Techniques du Languedoc \\ Centre d'Etudes d'Electronique des Solides, \\ Place Eugène Bataillon, 34060 Montpellier Cedex, France
}

(Reçu le 16 juillet 1975, accepté le 9 septembre 1975)

\begin{abstract}
Résumé. - Nous décrivons un appareillage électronique conçu pour commander le balayage en longueur d'onde d'un monochromateur à réseau, et qui peut être utilisé pour automatiser les variations d'un paramètre dans une gamme ajustable et prédéterminée, dans de nombreux cas expérimentaux et en particulier chaque fois que la valeur du paramètre est commandée mécaniquement. Utilisé avec un monochromateur à réseau linéaire, il permet de choisir la plage de balayage en longueur d'onde $\lambda_{1}, \lambda_{2}$ et le sens du balayage, d'enregistrer les variations de $\lambda$, de relever sur enregistreur XY les variations de la grandeur étudiée en fonction de $\lambda$, d'obtenir par lecture directe sur un voltmètre la valeur numérique de $\lambda$.
\end{abstract}

\begin{abstract}
An electronic apparatus which can be used to vary several experimental parameters automatically is described. This device has been used for monitoring a grating monochromator. It permits the wavelength range and the direction of variation of the wavelength $\lambda$ to be chosen, and the variations of the studied phenomenon with $\lambda$ to be recorded directly on a XY plotter ; it also gives the possibility of reading instantaneously the exact value of $\lambda$ on a digital voltmeter.
\end{abstract}

1. Introduction. - La plupart des ensembles expérimentaux actuels sont conçus de telle façon que l'expérimentateur soit à même de modifier aisément, d'une part les conditions de l'expérience, c'est-à-dire certains paramètres caractéristiques de la manipulation, comme par exemple la température, la pression, la longueur d'onde, d'autre part l'acquisition des données qui l'intéressent.

L'utilisateur peut ainsi, soit choisir des valeurs fixes du paramètre, soit, si l'étude le nécessite, se fixer une gamme programmée de variations, accompagnée d'une automatisation des mesures. Cette deuxième possibilité est de plus en plus utilisée, dans l'industrie comme dans les laboratoires de recherche, en particulier en optique quand il faut, pour étudier l'absorption, faire varier continuement la longueur d'onde. Le balayage en longueur d'onde et le relevé du spectre d'absorption sont alors automatisés.

Les spectromètres utilisés pour cela sont le plus souvent des spectromètres classiques du commerce [1], [2]. Plus récemment, en liaison avec le développement de la spectroscopie de modulation [3] les techniques d'étude ont évolué et les performances ont été sensiblement augmentées. L'automatisation de l'acquisition des données et la recherche de minimisation des erreurs ont été simultanément développées [4].

Ayant à effectuer des études de variations de poten- tiel de surface sous éclairement, nous avons été conduits à réaliser un ensemble simple, peu onéreux, permettant de faire varier la longueur d'onde $\lambda$ continuement et linéairement avec le temps entre deux limites $\lambda_{1}$ et $\lambda_{2}$ prédéterminées et affichées, de balayer la zone $\lambda_{1}, \lambda_{2}$ dans le sens désiré, de lire directement sur voltmètre numérique et d'enregistrer à chaque instant la valeur de $\lambda$.

L'ensemble électronique que nous avons réalisé et que nous présentons satisfait à ces critères. Il permet de plus de tracer directement sur enregistreur les variations de grandeur de l'effet étudié en fonction de la longueur d'onde dans la gamme $\lambda_{1} \lambda_{2}$ dont les limites sont choisies de façon à augmenter la précision de la mesure autour des points intéressants.

2. Principe. - Un dispositif $M$, mécanique ou électrique, permet de faire varier la grandeur $y$ dont on veut programmer les variations. Le principe de base du système réalisé suppose que l'on dispose d'une tension $V$ fonction monotone de $y(V=f(y))$. Les valeurs limites $V_{1}$ et $V_{2}$ correspondant aux frontières $y_{1}$ et $y_{2}$ de la plage de variation sont utilisées comme valeurs de consigne. Le système doit être réalisé de telle sorte que :

- si $y<y_{1}$, c'est-à-dire $V<V_{1}$, le dispositif $\mathrm{M}$ fait croître $y$ de façon à l'amener dans la plage $y_{1} y_{2}$; 
- si $y_{2}<y$, c'est-à-dire $V_{2}<V$, le dispositif $\mathrm{M}$ fait décroître $y$ de façon à l'amener dans la plage $y_{1}, y_{2}$; - si $y_{1}<y<y_{2}\left(V_{1}<V<V_{2}\right)$, le dispositif $\mathbf{M}$ impose à $y$ de balayer la plage $y_{1}, y_{2}$ dans le sens croissant ou décroissant suivant le cas.

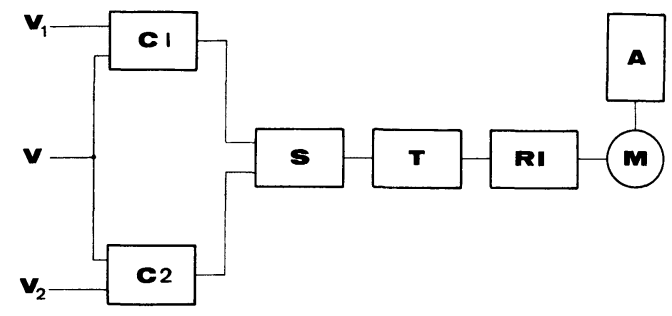

Fig. 1. - Schéma synoptique du circuit de commande de balayage : $\mathrm{C}_{1}, \mathrm{C}_{2}$ comparateurs; $\mathrm{S}$ sommateur; $\mathrm{T}$ trigger RI relais inverseur ; $\mathbf{M}$ moteur ; A alimentation moteur.

Le schéma synoptique représenté figure 1 correspond à un ensemble qui répond à ces impératifs. Il comprend :

- la source délivrant la tension de commande de $\mathbf{M}$,

- 2 comparateurs $\mathrm{C}_{1}$ et $\mathrm{C}_{2}$ permettant de comparer la tension $V$ respectivement aux valeurs de consigne $V_{1}$ et $\mathrm{V}_{2}$,

- un sommateur inverseur $\mathrm{S}$,

- un trigger $T$,

- un relais inverseur RI qui commande le dispositif $\mathbf{M}$, et plus précisément le sens de variation de la grandeur $y$. Ce relais est caractérisé par deux états 0 et 1 correspondant aux deux sens de variation possibles.

Désignons par $c_{1}, c_{2}, s, t, i$ les états logiques de sortie de $\mathrm{C}_{1}, \mathrm{C}_{2}, \mathrm{~S}, \mathrm{~T}, \mathrm{RI}$. Les comparateurs peuvent être dans deux états +1 ou $-1:\left(c_{1}, c_{2}= \pm 12 \mathrm{~V}\right)$, suivant que la tension $V$ est supérieure ou inférieure à là tension de consigne.

Le sommateur inverseur $\mathrm{S}$ de gain $\mathrm{G}$ effectue l'opé-

\begin{tabular}{cccr}
$\begin{array}{c}\text { Position } \\
-\end{array}$ & \multicolumn{1}{c}{$C_{1}$} & \multicolumn{1}{c}{$C_{2}$} & \multicolumn{1}{c}{$s$} \\
$V<V_{1}<V_{2}$ & -1 & -1 & +1 \\
$V_{1}<V<V_{2}$ & +1 & -1 & 0 \\
$V_{1}<V_{2}<V$ & +1 & +1 & -1 \\
$V_{1}<V<V_{2}$ & +1 & -1 & 0 \\
$V<V_{1}<V_{2}$ & -1 & -1 & +1
\end{tabular}

Sur ce tableau on constate que l'état numéroté 4 et l'état numéroté 0 sont identiques. Le système évolue donc de façon cyclique. Le même cycle aurait pu être décrit en prenant pour point de départ $V_{1}<V_{2}<V$ ou $V_{1}<V<V_{2}$. Toutefois, dans ce dernier cas, le sens d'évolution de $V$ dépend de l'état initial du trigger. Les états 1 et 3 du tableau sont en effet identiques à la valeur de $t$ près. Le montage définitif devra donc comporter un système permettant de positionner le ration $s=-G\left(c_{1}+c_{2}\right)$. Suivant la valeur de $c_{1}$ et $c_{2}$, donc de $V, s$ peut être dans les états logiques :

$$
\begin{aligned}
& s=0, \text { si }\left(V_{1}<V<V_{2}\right) \\
& s=1, \text { pour }\left(V<V_{1}<V_{2}\right) \\
& s=-1, \text { pour }\left(V_{1}<V_{2}<V\right) .
\end{aligned}
$$

Le trigger, caractérisé par deux états -1 et +1 , passe de l'un à l'autre quand le sommateur inverseur passe de l'état +1 à l'état -1 et réciproquement. Il commande alors le relais. Il ne change pas d'état lorsque le sommateur passe par l'état intermédiaire 0 .

3. Fonctionnement. - Une plage de variations $y_{1}$, $y_{2}$ ayant été choisie, on applique à l'entrée des comparateurs $C_{1}$ et $C_{2}$ des tensions respectivement égales à $V_{1}$ et $V_{2}$ et la tension $V$.

Supposons que $V<V_{1}<V_{2}$. Les comparateurs $\mathrm{C}_{1}$ et $\mathrm{C}_{2}$ sont alors dans l'état -1 . Le sommateur est dans l'état +1 , le trigger dans l'état -1 . Le relais est dans un état tel que $V$, donc $y$, évolue dans le sens des valeurs croissantes.

Lorsque $V$ dépasse la valeur $V_{1}$, le comparateur $\mathrm{C}_{1}$ change d'état, le sommateur vient dans l'état 0 . Le seuil du trigger n'est pas atteint. $V$ évolue toujours vers des valeurs croissantes.

Lorsque $V$ dépasse la valeur $V_{2}$, le comparateur $\mathrm{C}_{2}$ change à son tour d'état. Le sommateur vient dans l'état -1 , le trigger bascule à l'état +1 , faisant évoluer $V$ vers des valeurs décroissantes.

Lorsque $V$ repasse dans le domaine $V_{1}<V<V_{2}$, le comparateur $\mathrm{C}_{2}$ passe à l'état -1 , la sortie de $\mathrm{S}$ devient 0 , l'évolution se poursuit vers des valeurs décroissantes de $V$.

Lorsque enfin $V$ devient inférieur à $V_{1}, \mathrm{C}_{1}$ passe à l'état $-1, \mathrm{~S}$ passe à l'état +1 , le trigger bascule et impose à $V$ d'évoluer dans le sens des valeurs croissantes.

Le fonctionnement du système peut être résumé par le tableau suivant :

\begin{tabular}{ll}
\multicolumn{1}{l}{} & $i$ \\
-1 & - \\
-1 & 0 \\
+1 & 0 \\
+1 & 1 \\
-1 & 0
\end{tabular}

Evolution
$\quad$
$\lambda \uparrow$
$\lambda \uparrow$
$\lambda \downarrow$
$\lambda \downarrow$
$\lambda \uparrow$

No état
-
0
1
2
3
4

trigger de façon à choisir le sens initial d'évolution, dans le cas où $V_{1}<V<V_{2}$.

3.1 ElÉMENTS DE BASE DU MONTAGE. - Le montage réalisé a été conçu pour fournir à partir d'une source lumineuse et d'un monochromateur simple faisceau à réseau (Jobin et Yvon M 25) un faisceau lumineux dont la longueur d'onde $\lambda$ varie entre deux limites $\lambda_{1}$ et $\lambda_{2}$ prédéterminées. Le monochromateur 
utilisé permet de sélectionner la longueur d'onde $\lambda$ dont les variations sont fonction de la rotation $\theta \mathrm{du}$ réseau $\lambda=f(\theta)$. Commander les variations de $\lambda$ revient à commander la rotation du réseau ; nous avons pour cela fixé sur l'axe d'entraînement du réseau deux moteurs synchrones $M_{1}$ et $M_{2}$, un pour chaque sens de rotation. Un potentiomètre multitours monté sur le même axe nous permet d'obtenir une tension $V$ fonction de la longueur d'onde sélectionnée : $V=g(\lambda)$. A $\lambda_{1}$ et $\lambda_{2}$ correspondent les tensions de consigne $V_{1}=g\left(\lambda_{1}\right)$, $V_{2}=g\left(\lambda_{2}\right)$.

Ces tensions commandent un ensemble électronique constitué de quatre amplificateurs opérationnels type 741 , montés en comparateurs $\left(\mathrm{A}_{7}, \mathrm{~A}_{8}\right)$, sommateur $\left(A_{9}\right)$, trigger $\left(A_{10}\right)$ (Fig. 2$)$.

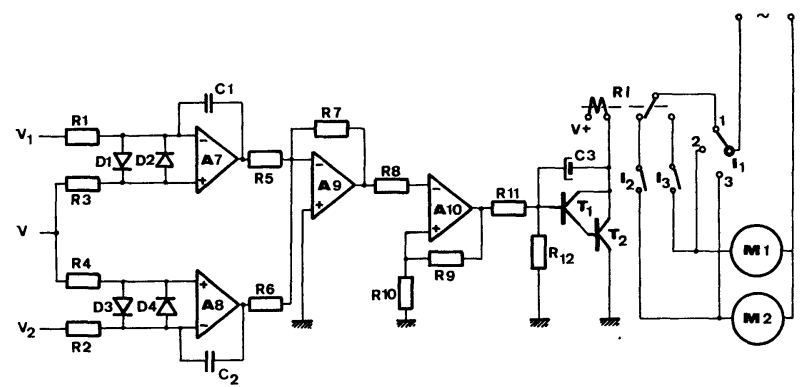

Fig. 2. - Schéma détaillé du circuit de balayage: $A_{7} A_{8}$ amplificateurs opérationnels type 741 , offset compensé ; $A_{9} A_{10}$ amplificateurs opérationnels type $741 ; \mathbf{R}_{1}, \mathbf{R}_{2}, \mathbf{R}_{3}$, $\mathbf{R}_{4}=27 \mathrm{k} \Omega 1 \% ; \mathbf{R}_{5}, \mathbf{R}_{6}=12 \mathrm{k} \Omega, \mathbf{R}_{7}=5,6 \mathrm{k} \Omega, \mathbf{R}_{8}, \mathbf{R}_{9}$, $R_{10}=33 \mathrm{k} \Omega, R_{11}=10 \mathrm{k} \Omega, \mathbf{R}_{12}=22 \mathrm{k} \Omega ; \mathrm{C}_{1}, \mathrm{C}_{2}=100 \mathrm{pF}$, $C_{3}=2,2 \mu F, D_{1}, D_{2}, D_{3}, D_{4}=1 \mathrm{~N} 914 ; T_{1}, T_{2}=2 \mathrm{~N} 3053$; $R I=$ relais inverseur $; M_{1}, M_{2}=$ moteur $; I_{1}$ : commutateur de choix de fonctionnement (1 automatique, 2 manuel marche $M_{1}, 3$ manuel marche $M_{2}$ ); $I_{2}, I_{3}$ fonctionnement automatique sélection d'un seul sens de balayage.

$V_{1}$ et $V_{2}$ sont appliquées sur les entrées inverseuses des comparateurs $C_{1}$ et $C_{2}$ dont les tensions de sortie, qui sont les tensions de saturation de l'amplificateur sont de $\pm 12 \mathrm{~V}$ environ. La tension $V$ est appliquée sur l'entrée commune non inverseuse. De façon à éviter tout risque d'oscillation parasite, ces comparateurs ont été contre-réactionnés par des condensateurs de $100 \mathrm{pF}$.

Le trigger a une tension de seuil élevée (6 V environ) pour augmenter la sécurité. Dans ces conditions, le gain du sommateur étant voisin de 0,5 sa tension de sortie est inférieure au seuil du trigger, donc équivalente à 0 , dans le cas où les comparateurs $C_{1}$ et $C_{2}$ sont dans des états opposés, même si les tensions de saturation de ces deux éléments n'ont pas la même valeur absolue. On voit que la sécurité est considérable, puisqu'il faut un décalage de tension supérieur à $12 \mathrm{~V}$ sur les entrées du sommateur, pour faire basculer le trigger. Le relais inverseur RI, alimenté par l'intermédiaire d'un transistor npn $2 \mathrm{~N} 3053$, commandé par le trigger, assure la mise sous tension de l'un ou l'autre des moteurs d'entraînement $\mathrm{du}$ réseau.

Le prépositionnement du trigger dans le cas où $V_{1}<V<V_{2}$ est effectué au moyen du commutateur
$I_{5}$ (Fig. 4) qui permet d'imposer temporairement la condition $V<V_{1}<V_{2}$ (évolution vers des $V$ croissants) ou la condition $V_{1}<V_{2}<V$ (évolution vers des $V$ décroissants).

3.2 Circuits DE MESURES ET RÉGlages. 3.2.1 Principe. - Le monochromateur utilisé permet de sélectionner une longueur d'onde $\lambda$ dont les variations en fonction de la rotation $\theta$ s'expriment par la relation $\lambda=A \theta+\lambda_{0}, A$ et $\lambda_{0}$ étant deux constantes caractéristiques du réseau. Le potentiomètre répéteur $P$ solidaire du réseau délivre une tension de la forme

$$
V=B \theta+V_{0} \text {. }
$$

La relation reliant $V$ à $\lambda$ est donc

$$
V=\frac{B}{A} \lambda-\frac{B}{A} \lambda_{0}+V_{0} .
$$

Un potentiomètre permet d'obtenir

$$
V=C\left(\frac{B}{A} \lambda-\frac{B}{A} \lambda_{0}+V_{0}\right)
$$

Cette expression peut être simplifiée par suppression du terme constant $C\left(V_{0}-\frac{B}{A} \lambda_{0}\right)$ et rendue aisément utilisable par un choix convenable du coefficient $B C / A$ dont la valeur doit pouvoir être ajustée aisément en fonction de l'appareillage et des critères expérimentaux.

Ce résultat est atteint à l'aide du montage représenté figure 3. Deux potentiomètres $P_{3}$ et $P_{4}$, alimentés par

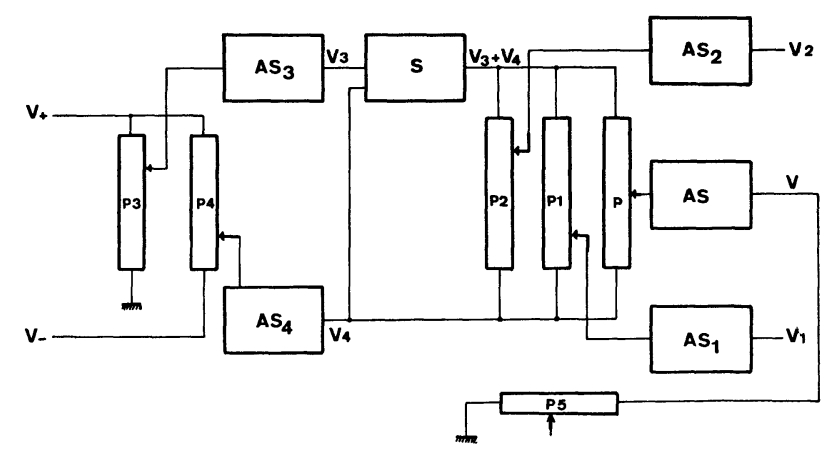

Fig. 3. - Schéma synoptique du circuit de mesure : AS, $\mathrm{AS}_{1}, \mathrm{AS}_{2}, \mathrm{AS}_{3}, \mathrm{AS}_{4}$ adaptateurs d'impédance; $\mathrm{P}$ potentiomètre répéteur ; $\mathbf{P}_{1}, \mathbf{P}_{\mathbf{2}}$ potentiomètres de choix des limites; $P_{3}$ potentiomètre de choix de la tension d'alimentation; $\mathbf{P}_{4}$ potentiomètre de décalage de zéro; $P_{5}$ potentiomètre de réglage du coefficient multiplicatif; S sommateur.

une source continue fournissent deux tensions réglables $V_{3}$ et $V_{4}$ répétées par deux amplificateurs suiveurs $\mathrm{AS}_{3}$ et $\mathrm{AS}_{4}$. Ces deux tensions sont ajoutées par le sommateur $\mathrm{S}$ qui délivre à sa sortie la tension $V_{3}+V_{4}$. La suppression du terme constant $C\left(V_{0}-\frac{B}{A} \lambda_{0}\right)$ impose d'ajuster $V_{0}$ de telle sorte que $V_{0}=\frac{B}{A} \lambda_{0}$, ce qui peut 
être réalisé par relèvement du potentiel $V_{4}$ du point bas d'alimentation des potentiomètres $\mathrm{P}_{1}, \mathrm{P}_{2}$ et $\mathrm{P}$ délivrant les tensions $V_{1}, V_{2}$ et $V$ répétées par les amplificateurs suiveurs $\mathrm{AS}_{1}, \mathrm{AS}_{2}$ et $\mathrm{AS}$.

Le potentiomètre $\mathrm{P}_{5}$ permet d'ajuster le terme $C$ de façon à ce que $B C / A$ prenne la valeur désirée et en particulier une valeur $10^{n}$, de façon à obtenir une lecture directe de $\lambda$.

Le schéma pratique de réalisation est représenté figure 4. Les amplificateurs suiveurs, le sommateur et l'inverseur ont été réalisés au moyen d'amplificateurs opérationnels du type 741 .

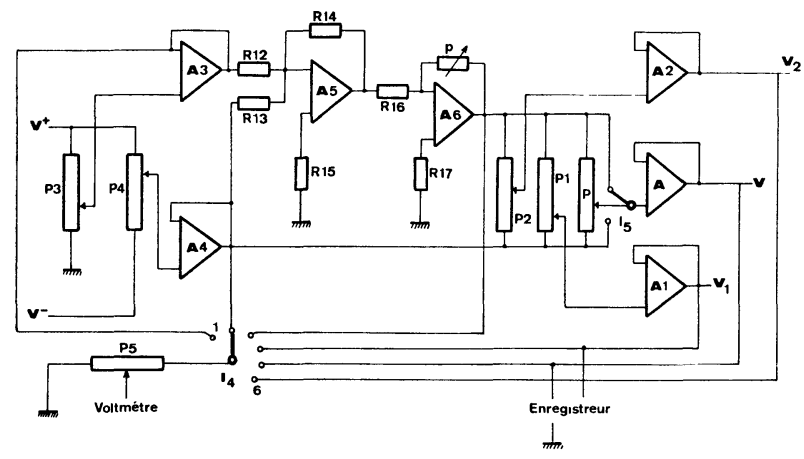

Fig. 4. - Schéma détaillé du circuit de mesure : A amplificateur opérationnel type 741 offset compensé ; $A_{1}, A_{2}, A_{3}, A_{4}$ amplificateurs opérationnels-type $741 ; \mathbf{P}, \mathbf{P}_{1}, \mathbf{P}_{2}, \mathbf{P}_{3}, \mathbf{P}_{4}, \mathbf{P}_{5}$ potentiomètres, p ajustable $50 \mathrm{k} \Omega ; R_{12}, R_{13}=27 \mathrm{k} \Omega 1 \%$ $\mathbf{R}_{14}=27 \mathrm{k} \Omega, \mathbf{R}_{15}=12 \mathrm{k} \Omega, \mathbf{R}_{16}=33 \mathrm{k} \Omega, \mathbf{R}_{17}=18 \mathrm{k} \Omega$; $I_{5}$ commutateur de sélection de mesure $\left(1-V_{3}, 2-V_{4}, 3-V_{3}+V_{4}\right.$, $\left.4-V_{1}, 5-V_{2}, 6-V\right)$.

$P_{1}, P_{2}$ et $P$ sont des potentiomètres linéaires 10 tours de valeur $47 \mathrm{k} \Omega . \mathrm{P}_{3}$ est un potentiomètre linéaire 1 tour de valeur $47 \mathrm{k} \Omega$. Les potentiomètres $\mathrm{P}_{4}$ et $\mathrm{P}_{5}$ qui doivent permettre un réglage fin sont constitués d'une résistance variable de 10 tours, de valeur $500 \Omega$ montée en série avec un potentiomètre 1 tour de $10 \mathrm{k} \Omega$.

Le commutateur $I_{5}$ permet d'aiguiller sur un voltmètre numérique les tensions $V_{1}, V_{2}, V_{3}, V_{4}, V_{3}+V_{4}$ ou $V$.

Les réglages sont effectués de la façon suivante : les valeurs de $V_{3}$ et $V_{4}$ étant quelconques, on sélectionne sucessivement au moyen d'un monochromateur deux longueurs d'onde $\lambda_{1}^{\prime}$ et $\lambda_{2}^{\prime}$ auxquelles correspondent deux valeurs $V_{1}^{\prime}$ et $V_{2}^{\prime}$ de la tension $V$ en sortie du potentiomètre $P$. On en déduit la valeur du terme $\frac{B}{A}=\frac{\mathrm{d} V}{\mathrm{~d} \lambda^{\prime}}$. Si le terme $V_{0}-\frac{B}{A} \lambda_{0}$ était nul, à la longueur d'onde $\lambda_{2}^{\prime}$ devrait donc correspondre la valeur $V_{2}^{\prime \prime}=\frac{B}{A} \lambda_{2}$. On doit donc ajuster la valeur de $V_{4}$ de telle sorte que cette condition soit remplie. Il ne reste plus alors qu'à diviser la valeur $V_{2}^{\prime \prime}$ par un coefficient constant, au moyen du potentiomètre $\mathrm{P}_{5}$ de façon à pouvoir lire $V_{2}^{\prime \prime}=10^{n} \lambda_{2}$.
3.2.2 Résultats. - Le système précédent peut être utilisé dans une grande gamme de variations de longueur d'onde. A titre d'exemple, nous avons choisi pour illustrer les résultats qu'on peut atteindre, une gamme de balayage comprise entre $\lambda_{1}=3500 \AA$ et $\lambda_{2}=4500 \AA$.

Pour étalonner le montage, nous avons choisi deux longueurs d'onde $\lambda_{1}^{\prime}=3656 \AA$ et $\lambda_{2}^{\prime}=4353 \AA$, correspondant à deux raies d'une lampe à vapeur de mercure OSRAM type HBO $75 \mathrm{~W}$ utilisée.

La courbe de variation expérimentale de l'intensité lumineuse du faisceau sélectionné par rotation du réseau, en fonction de la tension $V$ directement obtenue sur enregistreur est présentée figure 5 . On constate que

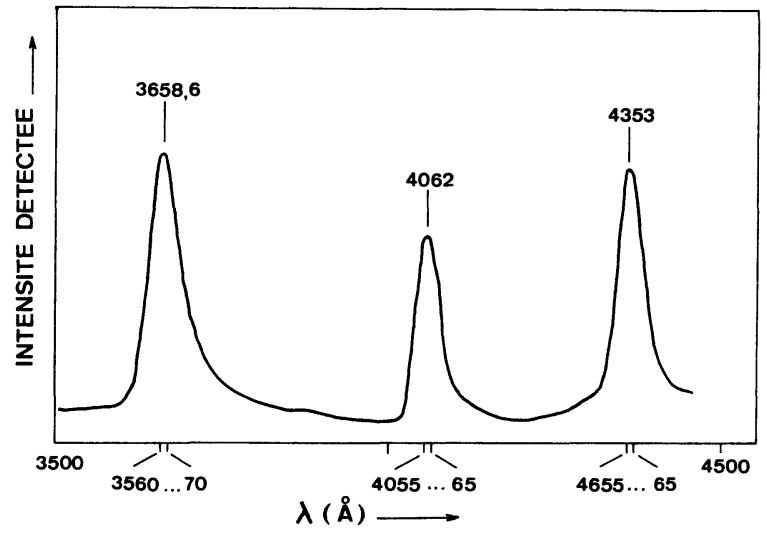

FIG. 5. - Intensité lumineuse fournie par une lampe à vapeur de mercure (OSRAM XBO $75 \mathrm{~W}$ ) en fonction de la longueur d'onde sélectionnée par un monochromateur (Jobin et Yvon, $M$ 25). Les valeurs lues au voltmètre numérique sont portées en abscisse. Les valeurs indiquées par le constructeur sont notées au-dessus des pics.

le pic de la lampe, situé d'après le constructeur à la longueur d'onde $\lambda_{3}^{\prime}=4062 \AA$ se trouve ici entre $4055 \AA$ et $4065 \AA$. On a donc une résolution meilleure que $10 \AA$ et une précision de l'ordre de $2 \%$.

La précision au niveau de la lecture dépend essentiellement de la linéarité du potentiomètre répéteur $P$, du système mécanique d'entraînement et du réglage de la tension $V_{3}$. La résolution est liée au potentiomètre répéteur. Ces deux quantités peuvent être améliorées, si l'expérimentation le nécessite, par l'utilisation d'un potentiomètre $P$ de meilleure qualité.

Signalons enfin qu'avec les éléments utilisés, la stabilité dans le temps est très satisfaisante, la dérive étant inférieure à $1 \%$ par heure.

4. Conclusion. - Le montage réalisé nous permet d'afficher numériquement et de façon simple les limites $\lambda_{1}$ et $\lambda_{2}$ de la plage de longueurs d'onde dans laquelle nous désirons utiliser le monochromateur à défilement linéaire. Ce réglage étant effectué, le système agit de façon à ce que la longueur d'onde sélectionnée vienne 
dans la zone $\lambda_{1}, \lambda_{2}$. Il impose alors l'exploration de cette zone par des aller-retours successifs ou par un balayage unique, dans un sens donné.

Dans tous les cas, quel que soit le type de réseau utilisé il autorise la lecture numérique instantanée de la longueur d'onde sélectionnée et rend possible l'enregistrement à pleine échelle sur un enregistreur XY, ce qui est particulièrement appréciable au voisinage des points singuliers intéressants.

Mis au point sur un cas particulier, ce montage peut être utilisé dans tous les cas où les variations d'un paramètre sont traduites par une variation de tension, c'està-dire dans de nombreuses manipulations de laboratoire.

\section{Bibliographie}

[1] KingsLake, Applied Optics and Optical Engineering, Optical Instruments Part II (Academie Press, New-York and London) 1969, vol. 5 .

[2] Bauman, Absorption Spectroscopy (John Willey and Sons, New-York London) 1962.

[3] Ricardo, Zucca, Shen, Y. R., Appl. Opt. 12 (1973) 1293.

[4] Robertson, A. R., Budde, W., MC Neely, F. T., Appl. Opt. 12 (1973) 2872. 